\title{
Trick and Please. A Mixed-Method Study On User Assumptions About the TikTok Algorithm
}

\author{
Daniel Klug \\ Carnegie Mellon University \\ Pittsburgh, Pennsylvania, USA \\ dklug@andrew.cmu.edu \\ Morgan Evans \\ Carnegie Mellon University \\ Pittsburgh, Pennsylvania, USA \\ morganev@andrew.cmu.edu
}

\author{
Yiluo Qin \\ Carnegie Mellon University \\ Pittsburgh, Pennsylvania, USA \\ yiluoq@andrew.cmu.edu \\ Geoff Kaufman \\ Carnegie Mellon University \\ Pittsburgh, Pennsylvania, USA \\ gfk@andrew.cmu.edu
}

\begin{abstract}
The short-form video sharing app TikTok is characterized by contentbased interactions that largely depend on individually customized video feeds curated by the app's recommendation algorithm. Algorithms are generally invisible mechanisms within socio-technical systems that can influence how we perceive online and offline reality, and how we interact with each other. Based on experiences from consuming and creating videos, users develop assumptions about how the TikTok algorithm might work, and about how to trick and please the algorithm to make their videos trend so it pushes them to other users' 'for you' pages. We conducted 28 qualitative interviews with TikTok users and identified three main criteria they assume influence the platform's algorithm: video engagement, posting time, and adding and piling up hashtags. We then collected 300,617 videos from the TikTok trending section and performed a series of data exploration and analysis to test these user assumption by determining criteria for trending videos. Our data analysis confirms that higher video engagement through comments, likes, and shares leads to a higher chance of the algorithm pushing a video to the trending section. We also find that posting videos at certain times increases the chances of it trending and reaching higher popularity. In contrast, the highly common assumption that using trending hashtags, algorithm related hashtags (e.g. \#fyp, \#foryou), and piling up trending hashtags would significantly push videos to the trending section was found not applicable. Our results contribute to existing research on user understanding of social media algorithms using TikTok as an example for a short-video app that is explicitly built around algorithmic content recommendation. Our results provide a broader perspective on user beliefs and behavior in the context of socio-technical systems and social media content creation and consumption.
\end{abstract}

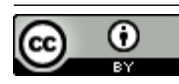

This work is licensed under a Creative Commons Attribution International 4.0 License.

WebSci '21, fune 21-25, 2021, Virtual Event, United Kingdom

(c) 2021 Copyright held by the owner/author(s).

ACM ISBN 978-1-4503-8330-1/21/06.

https://doi.org/10.1145/3447535.3462512

\section{CCS CONCEPTS}

-Information systems $\rightarrow$ Social networks; $\bullet$ Human-centered computing $\rightarrow$ Empirical studies in $\mathrm{HCI}$.

\section{KEYWORDS}

algorithm, user assumptions, TikTok, short-video app, qualitative interviews, hashtag analysis

\section{ACM Reference Format:}

Daniel Klug, Yiluo Qin, Morgan Evans, and Geoff Kaufman. 2021. Trick and Please. A Mixed-Method Study On User Assumptions About the TikTok Algorithm. In 13th ACM Web Science Conference 2021 (WebSci '21), June 2125, 2021, Virtual Event, United Kingdom. ACM, New York, NY, USA, 9 pages. https://doi.org/10.1145/3447535.3462512

\section{INTRODUCTION}

Short-video apps are distinct from video-sharing platforms (e.g. YouTube) and social networking services (e.g. Facebook, Instagram) [42] because they are exclusively designed for people to interact with and through user-generated video content [16] rather than with each other around it. After the discontinuation of Vine (20132017), the popularity of musical.ly (2014-2018) led to the recent increase in short-video apps, such as Byte, Triller, Zynn, or Likee, among which TikTok is undisputed in user numbers and user engagement worldwide ${ }^{1}$ and in the U.S. ${ }^{2}$ Sharing photos and videos online is one of the most popular activities among teenagers and young adults [41] who constitute the largest group of TikTok users in the U.S. ${ }^{3}$ TikTok videos are usually 15 to 60 seconds long and based on short music or sound files provided through the app [35]. TikTok video content is manifold, ranging from an experimental audiovisual playground [10], to entertaining dance content [32, 55], social activism [5,51], public health information [6], or celebrity content [1]. TikTok by default presents videos to the user on their 'for you' page as an endless [7], hard to anticipate [50] flow of auto-looped videos to swipe through. Standard social network characteristics (profile with user information, friends lists, tools to communicate) [9] are replaced by displaying the user's video content,

\footnotetext{
${ }^{1}$ https://datareportal.com/social-media-users?rq=tiktok [last accessed February 18, 2021]

${ }^{2}$ https://www.statista.com/statistics/1100836/number-of-us-tiktok-users [last accessed February 18, 2021]

${ }^{3}$ https://www.statista.com/statistics/1095186/tiktok-us-users-age [last accessed February 18, 2021]
} 
followers and likes, and by allowing commenting on videos. The content-based interaction on TikTok largely depends on the video curation by the app's recommendation algorithm [7]. Regarding video sharing platforms and apps, the algorithm of YouTube has been largely studied, for example, concerning inequalities in selecting content for feeds [8], recommendation patterns [2], the ranking of search results [45], or the catering of problematic content to keep audiences in a video viewing loop [12]. However, little research has looked at user experiences with or understanding of social media algorithms in relation to content creation. This user perspective is highly relevant for researching short-video apps as they are specifically designed around algorithms to motivate and retain users to consume and create large volumes of short-form video content. Because of the novelty of TikTok, research focusing on on users' understanding of its algorithm is especially scarce. Previously, the discontinued Vine app has been studied, for example, regarding popularity and lifetime of videos and follower-following relations [58]. Vandersmissen et al. (2014) found Vine videos received most likes and attention shortly after their creation and that sharing Vine videos on Twitter amassed significantly more likes [54].

In this paper we research assumptions about the TikTok algorithm based on user experiences from consuming and creating short-form videos and test user assumptions about how the algorithm apparently work against a data analysis of trending TikTok videos. We ask the following research questions:

- RQ1: What are users' experiences with and assumptions about how the TikTok algorithm works?

- RQ2: How do users' assumptions about the TikTok algorithm affect their video creation practices?

- RQ3: Do users' assumptions about the TikTok algorithm correspond with how the algorithm actually behaves?

\section{BACKGROUND AND RELATED WORK}

Algorithms are generally invisible mechanisms [24] within sociotechnical systems and digital infrastructures that can influence how we perceive the reality of everyday life online and offline [52], and how we interact with and in these realities and with each other [37,56]. This concerns, for example, results of search engines we use [23, 28], information we consume [4], and especially social interaction and self-presentation on social media platforms [20]. On social media platforms, algorithms present us with a digital identity [17] created from our online behavior, our content preferences, and the personal information we provide. This may appear as beneficial regarding social connectivity [53], visibility, and social power [13] in online contexts. However, algorithms can also present inherent biases by design and, for example, create and potentially reinforce social, political, or cultural stereotypes in our offline lifeworlds outside of digitally mediated contexts [14,29].

\subsection{How the TikTok Algorithm Most Likely Works}

Research on user theories about recommendation algorithms covers, for example, Facebook news feed curation [21, 22, 44], or Spotify music suggestions [49], and how user assumptions influence user engagement [15]. In contrast, so far only a few research studies have focused particularly on analyzing the TikTok algorithm
$[5,25,50]$ and only some information can be retrieved from blogs, journalistic resources, or press releases. Unlike Facebook, Instagram, or YouTube, and their short-video versions Instagram Reels and YouTube Shorts, TikTok does not generate video feeds based on content from accounts followed [25, 42]. Rather, the TikTok recommendation algorithm customizes video content for the individual user's 'for you' page [3] based on previous and continuous user engagement with presented video content through video viewing time, liking, commenting, and sharing. In addition, the 'for you' page feed is generated from videos that use trending hashtags or sounds [40]. In this way, by constantly learning users' video content preferences [50], the output of the TikTok algorithm can become visible to users [25] through the curated content feed that selects videos related to their apparent preferences. ByteDance, the company that owns TikTok, applies natural language processing to classify text elements and audio components in TikTok videos, and computer vision technology to automatically locate and categorize visual objects ${ }^{4}$. Together with analyzing hashtags and video captions, this information is seemingly used to evaluate a video for its selection by the recommendation algorithm [47]. New videos are initially shown to a small group of users who are likely to interact with the video and then shown to more users if the initial group favorably engages with the video [38]. This means, basically any video of any user may be pushed to a 'for you' page [38], and a 'for you' page may show very recent and highly popular videos alongside older ones or videos with only a few likes. According to TikTok $^{5}$, the algorithm mostly picks up on the sound, the hashtags, and the captions used in videos a user engaged with and recommends similar content. More experienced users share advice on how to take advantage of the TikTok algorithm by, for example, creating shorter videos, using trending sounds and hashtags, including teasers and questions [27], or posting at times when potential viewers are active [43].

\subsection{How Users Actually Experience the TikTok Algorithm}

User-centered qualitative studies of TikTok have mostly applied content analysis $[31,33,57]$ or cognitive walkthrough methods $[7,30]$ to reconstruct user experiences with the app. Only very few studies on TikTok (or its Chinese version, Douyin) have investigated users' understanding of or lay theories about the app algorithm. For example, Lu, Lu \& Liu (2020) found that some users stopped using Douyin because the algorithm apparently only provided homogenous content [36]. Simpson \& Semaan's (2021) interview study with LGBTQ+ TikTok users shows that they were aware of how the algorithm works and able to influence it to construct a desired 'for you' page feed, for example, by engaging with appealing content through hashtags [50]. Hence, TikTok users can be understood as produsers [11], meaning they are equally producers and users of content who observe and experience how the algorithm works for them.

\footnotetext{
${ }^{4}$ https://ailab.bytedance.com [last accessed February 18, 2021]

${ }^{5}$ https://newsroom.tiktok.com/en-us/how-tiktok-recommends-videos-for-you [last accessed February 18, 2021]
} 


\section{METHOD}

We followed a mixed-method approach to adequately address and answer [18] our research questions and to generate a complete and deep understanding of our research subject [19]. First, we conducted qualitative interviews with TikTok users on their video creation practices and assumptions about the TikTok algorithm. Second, based on interview results, we collected video meta data from the TikTok trending section to examine user assumptions about the algorithm through quantitative data analysis.

\subsection{Data Collection}

- Qualitative data collection: With IRB approval, we conducted 28 qualitative semi-structured interviews [34] in August 2020 with TikTok users in the U.S. We asked participants about general motivations and routines of consuming and creating TikTok videos, and specifically about their understanding of and experiences with the TikTok algorithm, and how these influence their video creation practices. Participants were aged 18 to 25 , and $65 \%$ were female. As of August 2020, they had between 300 and 450k followers, had posted between 15 and 425 videos, and had in total received between $10 \mathrm{k}$ and $11.5 \mathrm{~m}$ likes for their TikTok videos. We recruited participants through location-based and university related hashtags in TikTok videos (e.g. \#pittsburgh, \#pitt, \#upenn). We then messaged users who included their Instagram profile on TikTok through Instagram as TikTok does not allow messaging unless accounts are following each other. We messaged 192 users out of which 28 users participated in the interview study (14.5\% participation rate). Interviews were conducted by two experienced researchers and transcribed and anonymized using an online transcription service.

- Quantitative data collection: We used an open source scraping tool ${ }^{6}$ to collect video metadata information from the TikTok trending section through the TikTok API without logging into the app. This data collection is common [5] and useful for a general analysis because it is not based on a certain video phenomenon, music, or hashtag [48]. We collected 300,617 trending videos $(18,788$ on average per day) over 16 consecutive days from January 14th to 29th, 2021. The data was stored as csv-files with 34 data categories given in columns. Figure 1 shows the seven categories we used for our data analysis and for further data engineering.

- Data preprocessing: Prior to the quantitative data analysis, we used the Python function ast.literal_eval to convert each data entry in the 'hashtags' column from literal data into 'list' form so it could be properly processed by code. We then extracted the 'name' information for all hashtags from each list of all data entries and stored this data in a dictionary as key-value pairs (key = unique hashtag name; value = number of times the hashtag is being used by the videos). This dictionary is used for the hashtag hotness analysis and the unique hashtag analysis (see 4.3). We used a stop words library $^{7}$ to remove irrelevant words (e.g. "a", "is") from the hashtag list. In a second preprocess, we sorted and then by convention split the 'playCount' data column into two new groups: videos with the top $10 \%$ of play counts ("Top10\%") and videos with the remaining $90 \%$ of play counts ("Remaining90\%"). This allows to differentiate

\footnotetext{
${ }^{6}$ https://github.com/drawrowfly/tiktok-scraper [last accessed February 18, 2021] ${ }^{7}$ Natural Language Toolkit library: https://gist.github.com/sebleier/554280 [last accessed February 18, 2021]
}

our analysis of trending TikTok video data based on video plays on TikTok as de facto number of video views or video loops [38].

$\begin{array}{llll}\text { createTime } & 300617 & \text { non-null } & \text { int64 } \\ \text { videoMeta.duration } & 300617 \text { non-null } & \text { int64 } \\ \text { playCount } & 300617 \text { non-null } & \text { int64 } \\ \text { commentCount } & 300617 \text { non-null } & \text { int64 } \\ \text { diggCount } & 300617 \text { non-null } & \text { int64 } \\ \text { shareCount } & 300617 \text { non-null } & \text { int64 } \\ \text { hashtags } & 300617 \text { non-null } & \text { object }\end{array}$

Figure 1: The seven (out of 34) data columns of the generated csv-file that we used for our quantitative data analysis

\subsection{Data Analysis}

- Qualitative data analysis: In the qualitative analysis of the interview data, a team of three experienced researchers applied an open coding approach based on a code book as reference guide for the coding process. The code book creation followed a deductive approach in two steps. In the first step, all three researchers coded the first interview to generate an initial set of codes. All codes were then compared and showed a high agreement regarding the naming and description of all codes for similar interview statements and passages. This established approach for HCI and CSCW research [39] ensured validity of our qualitative results through agreement. Codes were then grouped into a system of higher and lower level codes in relation to what was asked in the interview questions [46]. Each researcher then used the code book to examine an equal number of the remaining 27 interviews. After the coding process, seven codes that were not applied were revisited and subsequently deleted from the code book. 29 codes that were only applied once were revisited, 16 codes were merged with more frequently applied similar codes, and 13 codes were kept as single applied codes. Following this process, we created 175 codes in 33 categories.

- Quantitative data analysis: In the statistical analysis, we used the video meta data collected from the TikTok trending section to examine three main user assumptions about how the TikTok algorithm picks up and pushes videos to the trending section that participants described in the interviews. First, we analyzed the video engagement for the collected TikTok videos by generating normalized pair plots to related number of comments ('commentCount'), number of likes ('diggCount'), and number of shares ('shareCount') to number of video play counts ('playCount'). Second, we ran a Two-Sample Kolmogorov-Smirnov test on the 'createTime' data to determine if trending videos with top $10 \%$ of play counts and remaining $90 \%$ significantly differ in their posting times. We converted the original time stamp from string of integers to Coordinated Universal Time (UTC $\pm 00: 00$ ) because the collected data did not include geographical or time zone data for the videos. Third, to measure the effects of adding and piling up hashtags, we generated the hashtag hotness based on the key-pair dictionary from the data preprocessing to describe the relationship between the total popularity of all hashtags added to a video and the number of play counts the video received. We used the MinMaxScaler function from Python scikit-learn library to scale the absolute play count of each hashtag 
per video from 0 to 1 ( 1 = hashtag with the highest number of play counts). We paired the scaled play count scores with the respective hashtags and then created a user defined function that locates all individual hashtags added to a video, assigns each hashtag the matching play count score, and sequentially creates an overall score for the hashtag hotness of a video. For example, a video with more frequently used hashtags will have a higher score. We then used the MinMaxScaler function to calculate the relative 'playCount' score of each video from 0 to 1 ( 1 = highest number of play counts). As a result, each video receives an overall scaled score for hashtag popularity, and an overall scaled score for play counts. Fourth, we performed a unique hashtag analysis. We first created two new key-value pair dictionaries for both trending videos with the top $10 \%$ and the remaining $90 \%$ of play counts (keys = unique hashtags; values $=$ number of videos that used the hashtag). We excluded all algorithm related hashtags (e.g. \#fyp, \#foryou) and analyzed the remaining hashtags added to the videos. We selected the 50 most used non-algorithm related hashtags, generated a double sided count ratio bar plot, and ran a Two-Sample Kolmogorov-Smirnov test to analyze their relative count ratio in the top $10 \%$ and the remaining 90\% group of trending videos (see Figure 6).

\section{RESULTS}

From our interview, we identify three main assumptions users think are decisive for a video to be picked up and pushed to 'for you' pages by the TikTok algorithm: video engagement, posting time, and adding trending and algorithm related hashtags and piling up hashtags. In each of the following sections (4.1, 4.2, 4.3), we will first discuss each assumption, and second describe our findings from testing the assumption through data analysis of a sample of trending TikTok videos.

In general, we find most participants tried to understand how the algorithm works to make their videos 'trend', and to reach either a larger or a specific audience's 'for you' pages: "I realized the algorithm is very interesting and weird. So it [the participants' video creation] started from like this idea of potential fame or clout or whatever" (P14). As video consumers, interviewees largely described favorable experiences with the TikTok algorithm. For example, they were aware that an algorithm caters to their 'for you' page because it showed and kept showing content they were interested in. Favorable experiences as TikTok consumers led them to expect the algorithm to be predictable in a similar way when creating and sharing videos. However, as video creators, interviewees had rather confusing experiences and primarily understood the TikTok algorithm as erratic without observable patterns of distributing videos: "Maybe a month into me using TikTok, all of my videos started to get pushed. And then it just dropped off. And so I was confused. I didn't know why that had happened" (P27).

\subsection{Video Engagement}

The first user assumption we identified from interviews is that high video engagement through comments increases the chances that the algorithm picks up on a video and helps to make it trend. As video creators, interviewees observed that high video engagement through likes, shares, and foremost comments seems to trigger the algorithm to pick up and push a video to 'for you' pages. Participants observed videos with a high play count tend to also have lots of comments: "I know like the more comments you get, the more likely other people already see your video. Cause I'm pretty sure that's how the algorithm works" (P4). This understanding is likewise based on their experience from creating and consuming TikTok videos. As a result, many interviewees said they try to induce user engagement in their videos, for example, by asking users to comment, to appeal to their understanding of video engagement and algorithmic video selection: "Like how do I get the most engagement out of this? I'm thinking little things I can do in saying like I have a cool phone case (...) like someone will comment on it or something like that" (P25).

In order to analyze this assumption for trending videos in general, we matched the number of comments ('commentCount'), the number of likes ('diggCount'), and the number of shares ('shareCount') for each video to its video plays ('playCount'). We generated pair plots and normalized all plotted data to properly display the correlations. We can see that all three measures positively correlate with each other (Figure 2). The upward correlations in the subplots indicate TikTok videos with a high number of play counts also have a high number of comments, likes, and shares, and vice versa. Therefore, we can validate the user assumption regarding video engagement across these variables for trending videos in general.

\subsection{Posting Times}

The second user assumption we found in interviews is that certain posting times would increase the chances for a higher number of video plays and hence for videos to become trending. Participants who were more experienced TikTok users mentioned they strategically post videos based on their assumption that they receive more play counts and engagement at certain times: "I will usually post or do a video the day before I post it. And then I'll wait till the next day around a good time that a lot of people are on TikTok and then I'll post it" (P5). This indicates the assumption of immediacy because participants believe algorithm activity directly relates to user activity and that the algorithm works to meet the content demand of active users. Therefore they believe posting a video when demand is apparently higher increases the chances of the algorithm picking up the video and pushing it to more active users. This can turn out to be beneficial: "If I filmed it later than like 6pm, I'll just post it the next day. Cause it will be too late to post" (P25). In contrast to the described immediacy, more senior TikTok users observed that it can take time for a video to gain play counts: "(...) sometimes the algorithm will work with you like a month later. Even like two days later off, you will get on the for you page and your video will start like gaining traction" (P3). This adds to the user experience of the algorithm being erratic as it is unclear how and when a video trends or gets pushed to 'for you' pages.

In our data analysis, we find a strong difference between posting times and video play counts from analyzing the 'createTime' data for trending videos within the top $10 \%$ of play counts and the remaining $90 \%$ (Figure 3). In general, we can see that trending videos within the remaining $90 \%$ of play counts were mostly posted between midnight to 4am UTC time, while trending videos within the top $10 \%$ of play counts were largely posted between $6 \mathrm{am}$ and $4 \mathrm{pm}$ UTC time. Most trending videos within the top $10 \%$ play counts were posted almost exactly opposite (11am UTC) to most of the trending 


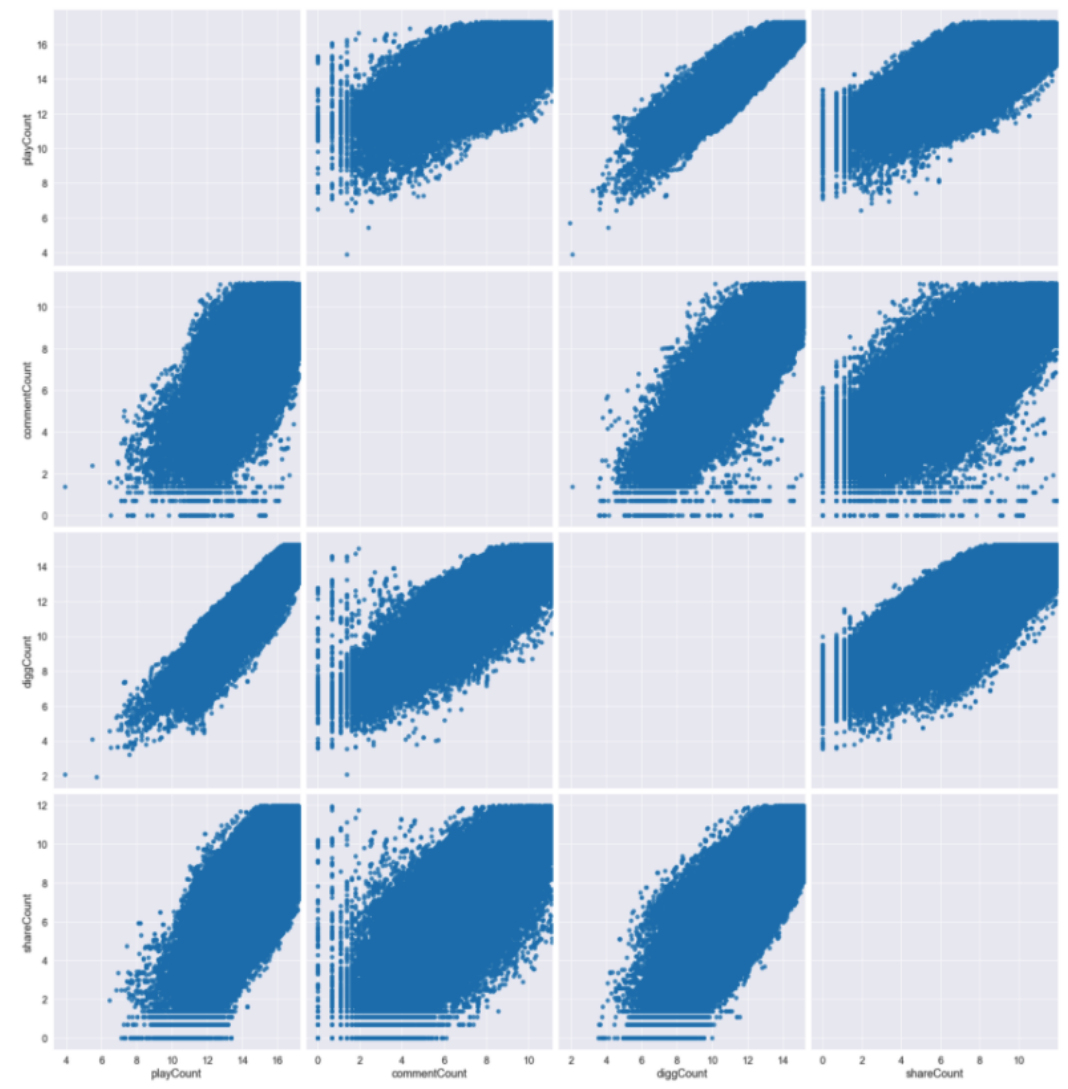

Figure 2: Correlations between number of video plays ('playCount'), number of comments ('commentCount'), number of likes ('diggCount'), and number of shares ('shareCount'). Each variable is crucial for defining the amount of video engagement on TikTok.

videos in the remaining $90 \%$ (1am UTC). We performed a TwoSample Kolmogorov-Smirnov test (significance level $=0.05$ ) to see if the two data samples come from the same distribution. Our result showed a significantly smaller p-value of $1.779 \mathrm{e}-41$. Therefore, we can validate this second user assumption and conclude that posting time played a significant role for a video to place within the top $10 \%$ or the remaining $90 \%$ of play counts within trending videos on TikTok.

\subsection{Adding and Piling Up Hashtags}

The third user assumption was that adding trending and algorithm related hashtags and piling up hashtags would increase the chance for a video to trend and to be pushed to 'for you' pages. Adding hashtags to TikTok videos is a common user practice to ensure visibility and reach within user communities $[6,26,42,50]$. We find interviewees assume hashtags are the main feature the algorithm picks up on to push videos to the trending section and a 'for you' page. Most participants added trending hashtags that TikTok suggested when creating a new video no matter if they actually relate to the video content or message, or themselves: "For the hashtags, I'll just do whatever hashtags are trending. So like when you click the hashtag there's this that automatically pop up. And normally I just click on those for my videos" (P4). We see participants assume trending hashtags to be suggestions by the app for content the algorithm will favor. Most users try to optimize their videos based on this assumption of a direct relation between trends and algorithmic distribution. The most common practice is adding hashtags, such as \#fyp or \#foryou, that are targeted at the TikTok recommendation algorithm and the video feed itself: "I found that using the hashtag FYP for you page (...) would also get more attention. So I always tried to use these hashtags in each video just so that it would pop up on people's feed" (P5). By adding algorithm related hashtags, users promote their video as appealing offer to the algorithm and indicate their goal of wanting to land on any 'for you' page: " $I$ always hashtag for you and for you page, just so you can get more like attention maybe" (P12). Interviewees also assumed it matters to pile up trending hashtags when creating a video because more hashtags would increase the value of a video for the algorithm and hence increase the chances of trending and being pushed to 'for you' pages: "I don't know if this is how the algorithm works or not but it seems like the more hashtags you have the more TikTok puts you on other people's screens" (P21).

To test user assumptions about piling up hashtags in our data analysis, we measured the hashtag hotness, that is, the relationship between the total popularity of all hashtags added to a video and 


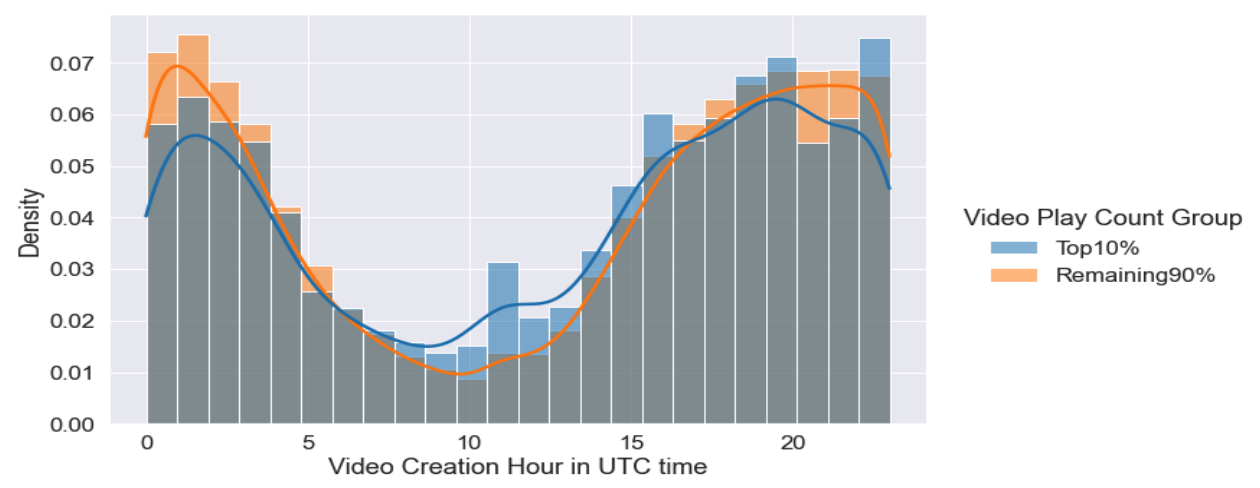

Figure 3: Distribution of trending videos per hour over 24 hours (in UTC time) based on analyzing the 'createTime' data. We can see strong differences between posting times and video play counts for trending videos within the top $10 \%$ (blue bars) and the remaining $90 \%$ group (orange bars).

the number of play counts the video received. We can find no obvious relationship between the total popularity of hashtags used in a single video and the number of play counts it received (Figure 4). Rather we see that a few videos with a very high relative hashtag hotness score received only very low play counts. We can conclude that the relative total popularity of added hashtags is not a main contributor to the play count of a video. This demonstrates that piling up trending hashtags does not automatically result in increased play counts; therefore, we can disprove this user assumption.

We also collected the 50 most used hashtags (excluding algorithm related, app related, and nonsense hashtags, such as \#xyzbca) to calculate the ratio count for each unique hashtag in the group of videos with top $10 \%$ play counts and remaining $90 \%$. The two-side bar plot (Figure 5) shows significant differences for the majority of hashtags. Many rather general hashtags (e.g. \#comedy, \#satisfying) are equally distributed in both groups, while hashtags predominantly in the top $10 \%$ videos seem to be related to seasonal phenomena (e.g. \#bye2020), TikTok stars (e.g. \#yzfamily), or recent viral phenomena (e.g. \#pudgywoke). To further test if the difference between the count ratio across all 50 hashtags in the two categories is significant, we performed a Two-Sample Kolmogorov-Smirnov test (significance level $=0.05$ ). The resulting $\mathrm{p}$-value $2.165 \mathrm{e}-23$ is significantly lower than the proposed significance level, which allows us to reject the null hypothesis that the two data samples came from the same distribution and allows to validate that they were intrinsically different.

Interview participants furthermore assumed that adding trending and algorithm related hashtags would increase the chance for a video to be picked up by the algorithm and to eventually make it to the trending section. While we cannot say how videos actually are selected, we found that there is a delay between trending hashtags suggested by TikTok and videos that are in the trending section on a certain day. We analyzed how many videos per day in our data set actually used at least one hashtag that was labeled as trending on that day to see if adding trending hashtags helps a video to get into the TikTok trending section. We found that some trending videos within the top $10 \%$ play counts were up to two weeks old at the time of our last data collection (January 29, 2021) (Figure 6). We can also see that videos in the top $10 \%$ group were generally older than in the remaining $90 \%$ group because the distribution is shifted left (Figure 6). This explains why almost none of the videos in the top $10 \%$ group used any of the daily trending hashtags.

Therefore, users have no direct way of ensuring that certain hashtags will make their video trend; they can only in retrospect assume the success of added hashtags. The strategy to add any suggested trending hashtag appears reasonable yet uncertain if users believe the TikTok algorithm recommends possible future trends.

\section{DISCUSSION AND FUTURE WORK}

Our interviews demonstrate that TikTok users are aware of the algorithm's functionality, both because of favorable experiences as consumers and rather questionable experiences as creators. They largely develop ideas and skills to trick and please the algorithm with the goal to optimize their experience as consumers and creators. Finding ways to explain and to attribute logical behavior to the TikTok algorithm is an essential part of consuming and creating videos.

From participants' descriptions, we identified three main assumptions (video engagement, posting time, adding and piling up hashtags) of how users think the TikTok algorithm picks up videos and pushes them to the trending section and to 'for you' pages. These assumptions influence users' video creation practices. For example, addressing viewers in a way that they would likely comment on a video, strategically waiting to post a video at a time when more users are apparently active, or foremost adding multiple hashtags that target trends or the algorithm instead of the video content. From analyzing a data sample of 300,617 trending TikTok videos, we find the user assumption that a high number of comments correlates with a high number of play counts (video engagement) to be true. We also find the assumption that posting videos at certain times significantly correlates with reaching the top $10 \%$ of play counts within the trending video section (posting times) to be true. However, we find the most common and most followed assumption that adding trending and algorithm related hashtags 


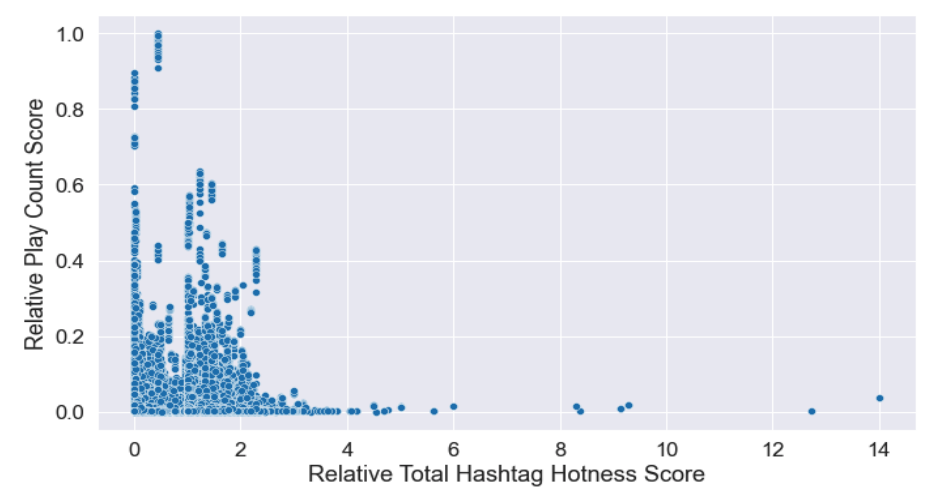

Figure 4: Relationship between Relative Hashtag Hotness Score and Relative Play Count. Each point represents a single video from the data set. We find no relationship between popularity of hashtags and received play counts. Videos with many hashtags rather have low play counts.

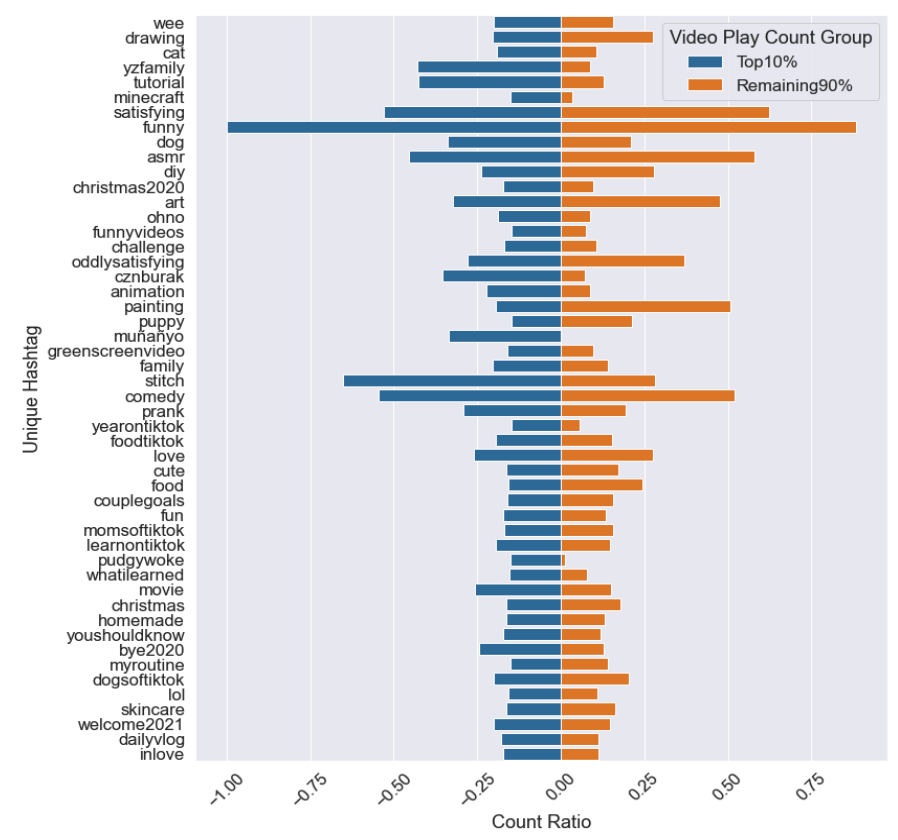

Figure 5: Distribution of the 50 most used hashtag non algorithm related hashtags based on hashtag count ration comparison. Blue bars indicate hashtags used by trending videos within top $10 \%$ play counts, orange bars indicate hashtags used by trending videos within the remaining $90 \%$.

and piling up hashtags would result in a video being pushed to the trending section to be not true.

Our study in general shows that it is quite common for TikTok users to evaluate app activity in order to estimate the behavior of the algorithm. Watching videos on their 'for you' page makes the algorithm visible for users through content, while measured video engagement (views, likes, shares, comments) visualize how the algorithm works for users as creators. Based on their favorable experiences as consumers, interviewees thought the algorithm should in the same way work for them and in their favor as creators. Ultimately, they hold the TikTok algorithm responsible for the success, reach, and virality of a video - rather than the style or content of a video. The goal of trying to understand the TikTok algorithm and to detect regularities and patterns in its behavior primarily serves to utilize the algorithm to optimize their TikTok experience as creators by either tricking or pleasing the algorithm. However, this seems to create a loop in which users react to the behavior of the algorithm, and the algorithm reacts to the behavior of users.

Interviewees also mentioned less common assumptions of how to increase the chances for TikTok videos to be picked up by the algorithm, such as choosing trending sounds and background music, 


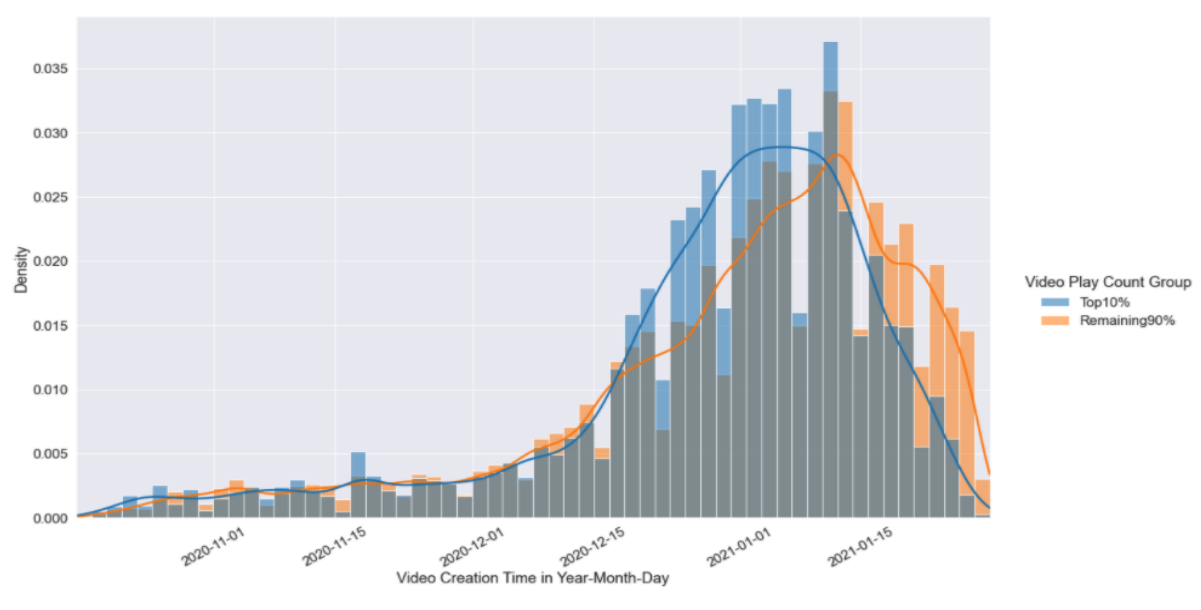

Figure 6: Comparison of original creation date (Year-Month-Day) for videos in the collected trending video data set on the last day of data collection (January 29th, 2021).

making shorter videos, speaking in short sentences, directly addressing the audience, or to not make videos montages. These criteria allow for future research on the intersection of user understanding, user practices, and algorithm analysis from socio-technological perspectives. Future qualitative research could focus on interviewing users about their video creation practices and how their understanding of the TikTok algorithm influences their self-presentation and the video content they create and share on TikTok. Based on our findings about how users experience the TikTok algorithm as consumers versus as creators, future research could use video observations or user diaries to examine how users interact with content on their individual 'for you' pages, and analyze how behavior might lead to changes in algorithmically generated content feed. TikTok is originally a music-based app; therefore, sounds and background music are very likely influencing if a video is trending. The video data we collected included metadata information on background music, future research can easily follow our approach to analyze the role of background music for videos to trend and to be pushed to 'for you' pages by the algorithm. The same applies to the factor of video length. Mixed-method approaches could also focus on qualitative content analysis of videos, added text elements, and video captions, and apply quantitative data analysis to examine which components boost the popularity of videos.

\section{LIMITATIONS}

We do not know exactly how the TikTok algorithm works and how videos are recommended. We understand 'trending' as a type of popular TikTok videos and as a quality that users aim for their videos to achieve. We also do not exactly know how videos actually end up in the trending section, and how many and which videos are labeled as 'trending' each day. Therefore, it is very likely that in our data collection videos were consistently scraped for consecutive days and put in the top $10 \%$ or the remaining $90 \%$ of play counts groups, and that results may not be generalizable. Collecting and analyzing other criteria and different types of TikTok videos may lead to different results.

\section{REFERENCES}

[1] Crystal Abidin. 2021. Mapping Internet Celebrity on TikTok: Exploring Attention Economies and Visibility Labours. Cultural Science fournal 12, 1 (2021), 77-103.

[2] Massimo Airoldi, Davide Beraldo, and Alessandro Gandini. 2016. Follow the algorithm: An exploratory investigation of music on YouTube. Poetics 57 (2016), $1-13$.

[3] Katie Elson Anderson. [n.d.]. Getting acquainted with social networks and apps: it is time to talk about TikTok. Library Hi Tech News 37 ([n. d.]).

[4] Jack Bandy and Nicholas Diakopoulos. 2020. Auditing News Curation Systems: A Case Study Examining Algorithmic and Editorial Logic in Apple News. ICWSM 14 (May 2020), 36-47.

[5] Jack Bandy and Nicholas Diakopoulos. 2020. \#TulsaFlop: A Case Study of Algorithmically-Influenced Collective Action on TikTok. (Dec. 2020). arXiv:2012.07716 [cs.HC]

[6] Corey H Basch, Grace C Hillyer, and Christie Jaime. 2020. COVID-19 on TikTok: harnessing an emerging social media platform to convey important public health messages. International journal of adolescent medicine and health 1 , ahead-of-print (2020).

[7] Aparajita Bhandari and Sara Bimo. 2020. TIKTOK AND THE "ALGORITHMIZED SELF”: A NEW MODEL OF ONLINE INTERACTION. AoIR Selected Papers of Internet Research (Oct. 2020).

[8] Sophie Bishop. 2018. Anxiety, panic and self-optimization: Inequalities and the YouTube algorithm. Convergence 24, 1 (2018), 69-84.

[9] danah boyd. 2010. Social network sites as networked publics: Affordances, dynamics, and implications. Networked self: Identity, community, and culture on social network sites (2010), 39-58.

[10] Ethan Bresnick. 2019. Intensified Play: Cinematic study of TikTok mobile app. Research Gate, available at: www. researchgate. net/publication/335570557_Intensified_Play_ Cinematic_study_of_TikTok_mobile_app (accessed 5 fanuary 2020) (2019).

[11] Axel Bruns. 2007. Produsage. In Proceedings of the 6th ACM SIGCHI Conference on Creativity \& Cognition. 99-106.

[12] Lauren Valentino Bryant. 2020. The YouTube Algorithm and the Alt-Right Filter Bubble. Open Information Science 4, 1 (2020), 85-90.

[13] Taina Bucher. 2012. Want to be on the top? Algorithmic power and the threat of invisibility on Facebook. New Media Soc. 14, 7 (Nov. 2012), 1164-1180.

[14] Jean Burgess and Ariadna Matamoros-Fernández. 2016. Mapping sociocultural controversies across digital media platforms: One week of\# gamergate on Twitter, YouTube, and Tumblr. Communication Research and Practice 2, 1 (2016), 79-96.

[15] Moira Burke, Cameron Marlow, and Thomas Lento. 2009. Feed me: motivating newcomer contribution in social network sites. In Proceedings of the SIGCHI conference on human factors in computing systems. 945-954.

[16] Zhuang Chen, Qian He, Zhifei Mao, Hwei-Ming Chung, and Sabita Maharjan. 2019. A study on the characteristics of douyin short videos and implications for edge caching. In Proceedings of the ACM Turing Celebration Conference-China. $1-6$.

[17] John Cheney-Lippold. 2018. We are data: Algorithms and the making of our digital selves. New York, NY: New York University Press.

[18] John W Creswell and Vicki L Plano Clark. 2017. Designing and conducting mixed methods research. Sage publications. 
[19] John W Creswell and Cheryl N Poth. 2016. Qualitative inquiry and research design: Choosing among five approaches. Sage publications.

[20] Michael A DeVito, Jeremy Birnholtz, Jeffery T Hancock, Megan French, and Sunny Liu. 2018. How people form folk theories of social media feeds and what it means for how we study self-presentation. In Proceedings of the $2018 \mathrm{CHI}$ conference on human factors in computing systems. 1-12.

[21] Motahhare Eslami, Karrie Karahalios, Christian Sandvig, Kristen Vaccaro, Aimee Rickman, Kevin Hamilton, and Alex Kirlik. 2016. First I" like" it, then I hide it: Folk Theories of Social Feeds. In Proceedings of the $2016 \mathrm{cHI}$ conference on human factors in computing systems. 2371-2382.

[22] Motahhare Eslami, Aimee Rickman, Kristen Vaccaro, Amirhossein Aleyasen, Andy Vuong, Karrie Karahalios, Kevin Hamilton, and Christian Sandvig. 2015. " I always assumed that I wasn't really that close to [her]" Reasoning about Invisible Algorithms in News Feeds. In Proceedings of the 33rd annual ACM conference on human factors in computing systems. 153-162.

[23] Ruoyuan Gao and Chirag Shah. 2020. Toward creating a fairer ranking in search engine results. Information Processing \& Management 57, 1 (2020), 102138.

[24] Tarleton Gillespie, Pablo J Boczkowski, and Kirsten A Foot. 2014. Media technologies: Essays on communication, materiality, and society. MIT Press.

[25] Benjamin Guinaudeau, Fabio Vottax, and Kevin Munger. 2020. Fifteen Seconds of Fame: TikTok and the Democratization of Mobile Video on Social Media. (2020).

[26] Shannon SC Herrick, Laura Hallward, and Lindsay R Duncan. 2020. "This is just how I cope": An inductive thematic analysis of eating disorder recovery content created and shared on TikTok using \#EDrecovery. International fournal of Eating Disorders (2020), 1-11.

[27] Henry Hien Ton. 2019. How to Crack the TikTok Algorithm to Create Viral Videos. https://medium.com/@henryhienton/how-to-crack-the-tiktokalgorithm-to-create-viral-videos-d8a00e38e5ae Last accessed February 18, 2021

[28] Lucas D Introna and Helen Nissenbaum. 2000. Shaping the Web: Why the politics of search engines matters. The information society 16, 3 (2000), 169-185.

[29] Matthew Kay, Cynthia Matuszek, and Sean A Munson. 2015. Unequal representation and gender stereotypes in image search results for occupations. In Proceedings of the 33rd Annual ACM Conference on Human Factors in Computing Systems. 3819-3828.

[30] David Bondy Valdovinos Kaye, Aleesha Rodriguez, and Patrik Wikstrom. 2020. YOU MADE THIS? I MADE THIS: CULTURES OF AUTOMATIC (MIS) ATTRIBUTION ON TIKTOK. AoIR Selected Papers of Internet Research (2020).

[31] Mona Khattab. 2020. Synching and performing: body (re)-presentation in the short video app TikTok. Wider Screen 21, 1-2 (2020).

[32] Daniel Klug. 2020. "It took me almost 30 minutes to practice this". Performance and Production Practices in Dance Challenge Videos on TikTok. arXiv preprint arXiv:2008.13040 (2020).

[33] Ioana Literat and Neta Kligler-Vilenchik. 2019. Youth collective political expression on social media: The role of affordances and memetic dimensions for voicing political views. New Media \& Society 21, 9 (2019), 1988-2009.

[34] Robyn Longhurst. 2003. Semi-structured interviews and focus groups. Key methods in geography 3, 2 (2003), 143-156.

[35] Xing Lu and Zhicong Lu. 2019. Fifteen seconds of fame: A qualitative study of Douyin, a short video sharing mobile application in China. In International Conference on human-computer interaction. Springer, 233-244.

[36] Xing Lu, Zhicong Lu, and Changqing Liu. 2020. Exploring TikTok Use and Non-use Practices and Experiences in China. In International Conference on Human-Computer Interaction. Springer, 57-70.

[37] Caitlin Lustig, Katie Pine, Bonnie Nardi, Lilly Irani, Min Kyung Lee, Dawn Nafus, and Christian Sandvig. 2016. Algorithmic authority: the ethics, politics, and economics of algorithms that interpret, decide, and manage. In Proceedings of the 2016 CHI Conference Extended Abstracts on Human Factors in Computing Systems. 1057-1062.

[38] Louise Matsakis. 2020. TikTok Finally Explains How the 'For You' Algorithm Works. https://www.wired.com/story/tiktok-finally-explains-for-youalgorithm-works/ Last accessed February 18, 2021.

[39] Nora McDonald, Sarita Schoenebeck, and Andrea Forte. 2019. Reliability and inter-rater reliability in qualitative research: Norms and guidelines for CSCW and HCI practice. Proceedings of the ACM on Human-Computer Interaction 3 , CSCW (2019), 1-23.

[40] Molly McGlew. 2020. This is How the TikTok Algorithm Works. https://later. com/blog/tiktok-algorithm/ Last accessed February 18, 2021.

[41] Sarah McRoberts, Haiwei Ma, Andrew Hall, and Svetlana Yarosh. 2017. Share first, save later: Performance of self through Snapchat stories. In Proceedings of the 2017 CHI conference on human factors in computing systems. 6902-6911.

[42] Juan Carlos Medina Serrano, Orestis Papakyriakopoulos, and Simon Hegelich. 2020. Dancing to the partisan beat: a first analysis of political communication on TikTok. In 12th ACM Conference on Web Science. 257-266.

[43] Masooma Memon. 2020. How the TikTok Algorithm Works in 2020 (and How to Work With It). https://blog.hootsuite.com/tiktok-algorithm/ Last accessed February 18, 2021

[44] Emilee Rader and Rebecca Gray. 2015. Understanding user beliefs about algorithmic curation in the Facebook news feed. In Proceedings of the 33rd annual ACM conference on human factors in computing systems. 173-182.

[45] Bernhard Rieder, Ariadna Matamoros-Fernández, and Ôscar Coromina. 2018. From ranking algorithms to 'ranking cultures' Investigating the modulation of visibility in YouTube search results. Convergence 24, 1 (2018), 50-68.

[46] Kathryn Roulston. 2014. Analysing interviews. The SAGE handbook of qualitative data analysis (2014), 297-312.

[47] Kayla Scanlon. 2020. The App That Knows You Better than You Know Yourself: An Analysis of the TikTok Algorithm. https://chatbotslife.com/the-appthat-knows-you-better-than-you-know-yourself-an-analysis-of-the-tiktokalgorithm-be12eefaab5a Last accessed February 18, 2021.

[48] Aliaksandra Shutsko. 2020. User-Generated Short Video Content in Social Media. A Case Study of TikTok. In International Conference on Human-Computer Interaction. Springer, 108-125.

[49] Ignacio Siles, Andrés Segura-Castillo, Ricardo Solís, and Mónica Sancho. 2020. Folk theories of algorithmic recommendations on Spotify: Enacting data assemblages in the global South. Big Data \& Society 7, 1 (2020), 2053951720923377.

[50] Ellen Simpson and Bryan Semaan. 2021. For You, or For" You"? Everyday LGBTQ+ Encounters with TikTok. Proceedings of the ACM on Human-Computer Interaction 4, CSCW3 (2021), 1-34.

[51] Sujatha Subramanian. 2021. Bahujan girls' anti-caste activism on TikTok. Feminist Media Studies 21, 1 (2021), 154-156.

[52] Emily van der Nagel. 2018. 'Networks that work too well': intervening in algorithmic connections. Media International Australia 168, 1 (2018), 81-92.

[53] José Van Dijck. 2013. 'You have one identity': Performing the self on Facebook and LinkedIn. Media, culture \& society 35, 2 (2013), 199-215.

[54] Baptist Vandersmissen, Fréderic Godin, Abhineshwar Tomar, Wesley De Neve, and Rik Van de Walle. 2014. The rise of mobile and social short-form video: an in-depth measurement study of vine. In Workshop on Social Multimedia and Storytelling, Vol. 1198. Citeseer, 1-10.

[55] Jorge Vázquez-Herrero, María-Cruz Negreira-Rey, and Xosé López-García. 2020. Let's dance the news! How the news media are adapting to the logic of TikTok. Journalism (2020), 1464884920969092.

[56] Michele Willson. 2017. Algorithms (and the) everyday. Information, Communication \& Society 20, 1 (2017), 137-150.

[57] Jing Zeng, Mike S Schäfer, and Joachim Allgaier. 2020. Reposting "till Albert Einstein is TikTok famous": The Memetic Construction of Science on TikTok. (2020).

[58] Lei Zhang, Feng Wang, and Jiangchuan Liu. 2014. Understand instant video clip sharing on mobile platforms: Twitter's vine as a case study. In Proceedings of Network and Operating System Support on Digital Audio and Video Workshop. $85-90$. 